\title{
OC11 - Social support and intervention focus to minimize the effects of oncological disease in healthy siblings
}

Goreti Marques (Portugal)ํㅜㄹ Beatriz Araujo (Portugal)²

${ }^{1}$ Escola Superior de Enfermagem de Santa Maria do Porto; ${ }^{2}$ Universidade Católica do Porto

Theme: Complex health care and chronic disease management.

Keywords: Child nursing, family, impact of oncological disease in healthy siblings; social support.

Introduction: The entire process underlying cancer disease not only affects the sick child, but also the healthy siblings, which should be the centre of attention from parents and nurses.

Objectives: To identify the impact of the disease in healthy siblings and know the importance of social support to minimise its impact in healthy siblings.

Methodology: Descriptive, correlational study, a sample of 128 families of children with oncological diseases, between August 2011 and January 2013. In order to do our research we used the following tools: Assessment questionnaire of the children's oncological diseases impact on the family and Satisfaction Scale Social Support.

Results: Families with a higher impact on family structure and more accrued expenses showed greater impact of the disease in healthy siblings, the most satisfied with their social support showed less improvement.

Conclusions: Nurses should seek interventions that increase the perception of the need for social support of families.

\section{OC12 - A journey suspended in time: parents' experiences of caring for their child who is 'technology dependent' with complex ongoing healthcare needs at home}

Yvonne Corcoran (Ireland) ${ }^{1}$; Prof Owen Barr (United Kingdom)²; Dr Rosario Baxter (United Kingdom)²

${ }^{1}$ Dublin City University; ${ }^{2}$ Ulster University

Theme: Complex health care and chronic disease management.

Keywords: Caregiver, complex care, parents, technology-dependent children.

Introduction: Increasing numbers of children with complex healthcare needs who are also dependent on medical technology are surviving and being cared for at home by their parents. 
Aim: To gain an in-depth understanding of the lives of parents and the impact of their caregiver role on their family when they care for their child at home.

Method: An interpretative phenomenological design was deployed to interpret the narratives of sixteen parents through interviews and digital audio diaries.

Discussion: The complex journey parents undertake as caregivers to their child at home.

Conclusion: The understanding revealed will prompt practitioners to reflect on how to promote best practice for this group of children, their parents and their families by recognising the complexity of the care parents provide for their child and the support required in order to sustain their caregiver role at home.

\section{OC13 - Parents' support needs during and following the death of their baby}

Michaela Barnard (United Kingdom) ${ }^{1}$

\section{${ }^{1}$ University of Salford}

Theme: Complex health care and chronic disease management.

Keywords: End of life, grief, neonatal, parents, support.

Introduction: Support for bereaved parents after the death of a baby in the neonatal period is variable in the UK.

Aim: To explore parents' experiences and perceptions of support needs during and following the death of their baby.

Methods: An interpretative phenomenological study using unstructured interviews was undertaken. Analysis was undertaken using interpretative phenomenological analysis.

Results: Parental grief journeys are complicated by events experienced at the end of their baby's life. A variety of support systems are needed to facilitate parental grief journeys.

Discussion: The variation in availability of support for parents after a neonatal death needs addressing. Whilst parental need is individualised, most parents need support in some format.

Conclusions: Health professionals have a key role to play in supporting parents and signposting other supportive mechanisms after a neonatal death.

\section{OC14 - Implementation of an educational program in children's post - operative pain}

Fotini Mavridi (Greece) ${ }^{1}$; Anastasia Statiri (Greece) ${ }^{2}$;

Adelais Tsiotou (Greece)³; Evangelia Kaliardou (Greece); Anastasia Doxara (Greece) ${ }^{1}$; Vasiliki Matziou (Greece)²

${ }^{1}$ Surgical Ward, 'P\&A Kyriakou' Children's Hospital, Athens; ${ }^{2}$ National \& Kapodistrian University of Athens, Nursing Department, Athens; ${ }^{3}$ Department of Anaesthesiology, 'P\&A Kyriakou' Children's Hospital, Athens 